\title{
Heart and Lung Mechanical Assist Devices are Comprehensively Tested by the New Hybrid Simulators
}

\author{
M. Darowski ${ }^{1}$, K. J. Pałko ${ }^{1}$, M. Kozarski ${ }^{1}$, K. Zieliński ${ }^{1}$, K. Górczyńska ${ }^{1}$, G. Ferrari ${ }^{2}$, \\ L. Fresiello², A. Di Molfetta ${ }^{2}$ \\ ${ }^{1}$ Nałęcz Institute of Biocybernetics and Biomedical Engineering, PAS, Ks. Trojdena 4, 02-109, Warsaw, Poland \\ ${ }^{2}$ Institute of Clinical Physiology, Section of Rome, CNR, Via San Martino della Battaglia 44, 00185, Rome, Italy \\ Email:mdar@ibib.waw.pl
}

Received 2012

\begin{abstract}
Two applications of the hybrid simulators have been presented as examples: nonpulsatile VAD interaction with lumped parameters cardiovascular system numerical model and respirator interacting with the Dubois numerical model of obstructive lung diseases. The results of simulations showed how the tested assist devices change biological system - assist device characteristics in the course of heart or lungs diseases and how it influences pressure and flow in a particular point of cardiovascular or respiratory system numerical model.
\end{abstract}

Keywords: Ventricular Assist Device; Respirator; Modelling; Hybrid Simulator

\section{Introduction}

Different type of heart and lung support systems and devices like ventricular assist devices (VAD) and respirators have been developed up to now but the problem how to test them, objectively, compare their features - advantages and disadvantages is still to be solved [1-3].

The heart and lung assist devices are, generally speaking, mechanical pumps that should be tested before their clinical use, according to appropriate standards. In order to access the features of these devices it is necessary to connect them to mechanical models of the biological organ or system (the human circulatory or respiratory systems) that are to be assisted or supported. Such mechanical models of the human circulatory or respiratory systems are expensive, as they have to be complex to simulate different pathologies of the biological (cardiovascular or respiratory) systems.

Computer models of the human cardiovascular or respiratory systems are much cheaper than the mechanical ones but, on the other hand, they can not be connected with mechanical assist devices like blood pumps or respirators $[4,5]$.

\section{Materials and Methods}

The solution we propose to test heart and lung assist devices is to use a new type of simulators (patent pending) - so called hybrid ones. They consist of a computer model of the human cardiovascular or respiratory system and a specially designed interface to connect this model with the mechanical assist device. These interfaces (electro-hydraulic for VAD or electro-pneumatic for respirators) play a role of impedance converters, as they enable to convert a part of the numerical model into a physical part of the same impedance, to which the tested assist device is connected (Figure 1).

It was possible to realize this concept thanks to the applica- tion of electrically connected flow and/or pressure sources as basic components of the impedance converters (see Figures 2-3).

There are varieties of technical realization of the impedance converter. In a case of testing the VAD we used a gear pump to build the voltage controlled flow source being a crucial part of the impedance converter (Figure 2) and in a case of testing the respirators, a voltage controlled piston flow source was used as a basic component of the impedance converter (Figure 3).

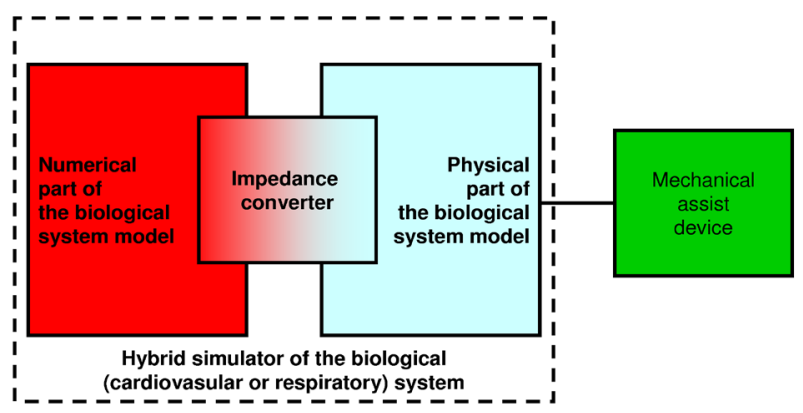

Figure 1. The idea of testing mechanical assist devices on a hybrid simulator of the cardiovascular or respiratory system.

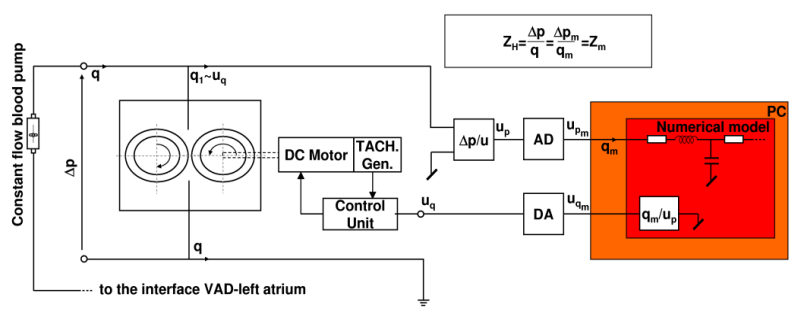

Figure 2. Set-up of the hydro-numerical interface (VAD-left atrium) for testing a nonpulsatile VAD on the hybrid simulator with a gear pump as a voltage controlled flow source. 


\section{Results}

Two applications of the mentioned above simulators have been presented as examples: nonpulsatile VAD interaction with lumped parameters cardiovascular system numerical model and respirator interacting with the Dubois numerical model of obstructive lung diseases. The results of simulations showed how the tested assist devices change biological systems characteristics in the course of heart or lungs diseases and how it influences pressure and flow in a particular point of cardiovascular or respiratory system numerical model.

The results of simulations of the cardiovascular system interacting with a nonpulsatile VAD were shown in Figure $\mathbf{4}$ as P-V loops.

Modelling of physiological and pathological states of the cardiovascular system was done and in the case of pathology the VAD was applied as assist 1 and assist 2 .

As we can see from Figure 4, VAD application changes P-V loop location but not so dramatically as pathology of the cardiovascular system.

The results of simulations of the respiratory system interacting with a respirator are shown in Figure 5 as time curves of control (Pin) and pleural (Pt) pressures. The respirator is providing pressure control ventilation of the human lungs which is a typical Dubois model with an abstractive lung disease. As can be seen from Figure 5, this disease causes positive end expiratory pressure to appear in the lungs.

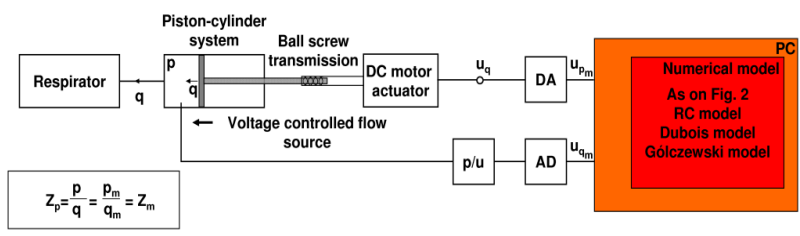

Figure 3. Set-up for testing a respirator on the hybrid simulator with a voltage controlled piston flow source.

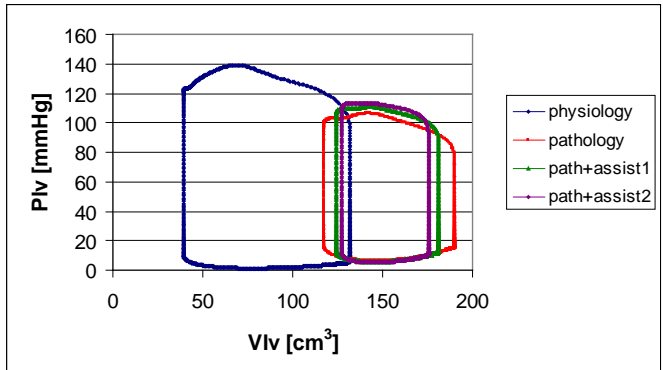

Figure 4. The results of simulations of the cardiovascular system interacting with a nonpulsatile VAD.

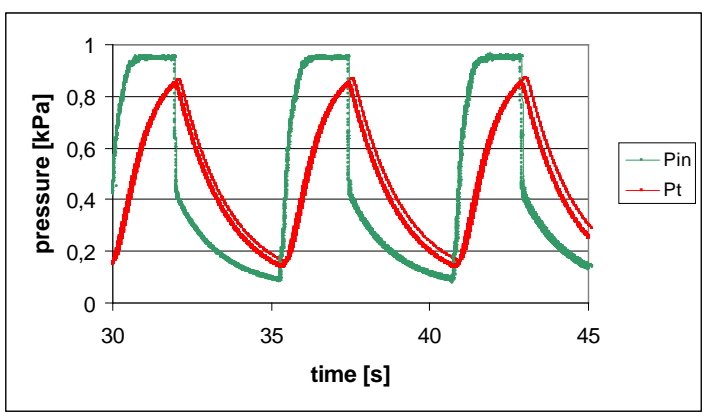

Figure 5. The results of simulations of the respiratory system interacting with a respirator.

\section{Conclusion}

Tests of the hybrid simulators performance and simulations of their interaction with heart and lung assist devices proved that they are reliable and accurate tools to test these devices and help to optimize their applications in different cases of heart and lungs pathology (as decision support systems).

\section{Acknowledgements}

The research leading to these results has received funding from the European Community's Seventh Framework Programme (FP7/2007-2013) under grant agreement No. 248763 (SensorART Project).

\section{REFERENCES}

[1] M. S. Slaughter, J.G. Rogers et al., "Advanced Heart Failure Treated with Continuous- Flow Left Ventricular Assist Device”, N. Engel. J. Med., vol. 361, pp. 2241-2251, December 2009.

[2] K. Górczyńska, "Circulatory Assistance: Basic Classification of Heart Assistance Methods and Devices", Biocybern. and Biomed. Eng., vol. 31, pp. 3-15, January 2011.

[3] S. Garcia, F. Kandar, A. Boyle, M. Colvin-Adams et al., "Effect of Pulsatile and Continuous -flow Left Ventricular Assist Devices on Left Ventricular Unloading”, J Heart and Lung Trans., vol. 22, pp. 261-267, March 2008.

[4] D. Morley, K. Litwak, P. Ferber, P. Spence, R. Dowling, B. Meyns et al., "Hemodynamic effects of partial ventricular support in chronic heart failure: Results of simulation validated with in vivo data”, J. Thorac. Cardiol. Surg., vol. 133, pp. 21-28, January 2007.

[5] K.W. Gwak, B.E. Paden, J.F. Antaki, I.S. Ahn, "Experimental Verification of the Feasibility of the Cardiovascular Impedance Simulator”, IEEE Trans Biomed Eng, vol. 57, pp. 1176-83, May 2010. 